\title{
Magnetic fields of pulsars surrounded by accretion disks of finite extension
}

\author{
N. R. Sibgatullin ${ }^{1,2}$, I. N. Sibgatullin ${ }^{1,3}$, A. A. Garcia ${ }^{1}$, and V. S. Manko ${ }^{1}$ \\ ${ }^{1}$ Departamento de Física, Centro de Investigación y de Estudios Avanzados del Instituto Politécnico Nacional, AP 14-740, \\ 07000 México DF, Mexico \\ e-mail: [sibgatullin; aagarcia;vsmanko]@fis.cinvestav.mx \\ 2 Faculty of Mechanics and Mathematics, Moscow State University, Moscow 119899, Russia \\ e-mail: sibgat@mech.math.msu.su \\ 3 Institute of Mechanics, Moscow State University, Moscow 119192, Russia \\ e-mail: sibgat@imec.msu.ru
}

Received 11 November 2003 / Accepted 29 March 2004

\begin{abstract}
The problem of finding the magnetic field of a system consisting of a magnetized neutron star and a perfectly conducting accretion disk of finite extension is reduced to the solution of the integral Fredholm equation of the second kind. Unlike the pioneering works of Aly (1980), Riffert (1980), we admit that an arbitrary total electric current can flow in the disk with finite outer rim. Having regularized the Fredholm equation, we obtain a convenient method for the construction of the magnetic field. The distribution of the magnetic field along the disk is plotted for different values of the total current and the ratios between the outer and inner radii of the disk.
\end{abstract}

Key words. accretion, accretion disks - magnetic fields

\section{Introduction}

The radiation of pulsars in close binary systems is now believed to be caused by the accretion of matter from accretion disks. A thin accretion disk can be stable due to the transport of angular momentum by the accreting matter (Pringle \& Rees 1972; Shakura \& Sunyaev 1973).

When a neutron star possesses weak magnetic fields $\left(B_{\text {surf }}<10^{8}\right)$, the accreting matter approaches the stellar surface. A difference between the Keplerian frequency and the pulsar's angular velocity may result in Kelvin-Helmholtz instabilities. Then a boundary layer is formed which gives rise to a strong X-ray radiation. An estimation of the energy of this radiation from the boundary layer and from the accretion disk was given within the framework of the general theory of relativity by Sibgatullin \& Sunyaev (2000a,b). Inogamov \& Sunyaev (1999) worked out the theory of a spreading layer on the neutron star surface.

For neutron stars with strong magnetic fields ( $B_{\text {surf }} \geq$ $10^{12}$ Gauss) the stellar magnetic field pressure is balanced in the magnetosphere by the pressure of the accreting matter. Near the magnetosphere, the magnetic field of the pulsar penetrates the accretion disk and causes the matter to rotate rigidly together with the pulsar. The turbulent and magnetic viscosities play a dominant role in the disk evolution. For the shear component $W$ of the stress tensor, Shakura \& Sunyaev (1973) suggested the relation $W=\alpha \rho c_{\mathrm{s}}^{2}$, where $\rho$ is the local average density of the accreting matter, and $c_{\mathrm{S}}$ is the local sound velocity. They constructed the standard disk model and established the dependence of the main parameters of the disk (such as pressure, thickness, density, radial accretion velocity, etc.) on the distance from the pulsar. The heat produced due to viscous dissipation of energy in the disk is fully emitted from the sides of the disk in the Shakura-Sunyaev model. In the Narayan model of an advective disk (see for instance Popham \& Narayan 1995) the heat is partially carried away by the accreting matter.

If the small-scale magnetic field turbulence inside the disk is sufficiently strong, then the effect of its reconnection with a weak exterior magnetic field may cause the penetration of the latter inside the disk, leading to the appearance of an effective conductivity $\sigma_{\text {eff }}$ (Ghosh \& Lamb 1979). In this case, in addition to the viscous dissipation of energy, there also arises an electric current dissipation $i^{2} / \sigma_{\text {eff }}, i$ being the local electric current. Another situation was considered by Scharlemann (1978). For a sufficiently weak small-scale magnetic field turbulence inside the disk, the surface currents in the disk screen the exterior magnetic field because of the high conductivity of matter in the disk. This gives rise to the proper magnetic field of the disk which is superposed on the dipole magnetic field of the pulsar. To estimate the magnetospheric radius in the disk's plane, one needs to use the combined magnetic field near the interior rim of the disk. It should be mentioned that there is a large 
number of instabilities in the turbulent plasma of the accretion disk (Scharlemann 1978; Ghosh \& Lamb 1979; Ichimaru 1978; Börner 1980; Anzer \& Boerner 1980). However, a consistent theory of the capture of matter by the magnetic field lines along which the matter moves toward the magnetic poles of the pulsar has not yet been constructed.

The radius of the inner rim can be found from the condition of counterbalance of the magnetic field of the pulsar and the magnetic field of the accretion disk. This led to the development of certain model representations for the magnetic fields of pulsars involving the radius of the inner rim of the accretion disk as a free parameter. The distance from the pulsar at which the magnetic field stops the accretion is much greater than the dimensions of the pulsar, so the magnetic dipole model is a good approximation for such distances.

The magnetic field of a pulsar induces local electric currents in the diamagnetic disk. Hence the normal component of the magnetic strength must vanish on the disk. The general case in which the axis of rotation and the magnetic dipole axis are not aligned is reduced to the superposition of the following two solutions:

(i) The solution with aligned axes of rotation and magnetic dipole.

(ii) The solution with the axis of the magnetic dipole lying in the plane of the accretion disk.

This important problem was first considered by Lipunov (1978) for the plane approximation and an infinite accretion disk. Somov et al. (2003) in the plane case have made an ingenious attempt to take into account the pressure of the interstellar gas and the deformation of the infinite disk.

In the more realistic axially symmetric case Aly (1980) obtained an exact solution for a magnetic dipole confined by an infinite thin disk (see also Kundt \& Robnik 1980, who gave the plots of magnetic lines in the case of non-aligned axes of magnetic dipole and rotation). In fact, these solutions could be obtained from the well-known solutions for the flow of an ideal fluid past the disk by application of Thompson's transform (Sibgatullin \& Doroshenko 2002). They correspond to the case where there is no azimuthal current flowing in the accretion disk. Riffert (1980) considered a more general case for a magnetic dipole confined by an infinite thin disk with an arbitrary constant which describes the amount of magnetic flux though the hole in the middle of he disk.

From the physical point of view the condition of perfect conductivity can be fulfilled only within a specific distance of the magnetized neutron star, or the accretion disk itself should be considered as finite.

In the present paper we shall treat the case of the combined dipole-disk system in which the accretion disk is supposed to be finite (a plane ring) and to possess an arbitrary circulation of the magnetic field around it. According to Ampere's theorem, the existence of the circulation is explained by a nonzero total azimuthal current in the disk. In this case the problem is far more complicated than in the case of an infinite disk. We will show that it can be formulated in terms of the so called triple integral equations (see some approaches for electrostatic plane rings of finite extension in the monograph of Sneddon 1966).
We have succeeded in reducing the problem to the solution of the integral Fredholm equation of the second kind containing an arbitrary constant which is expressible through the circulation. This work is restricted to the case of aligned axes of rotation and magnetic dipole. A numerical investigation of this equation in particular has permitted us to obtain the distribution of the magnetic field strength along the disk radius and to obtain magnetic surfaces in all space.

\section{Boundary conditions and Fredholm equation}

As follows from Maxwell's equations, the magnetic field is potential outside the sources in the thin accretion disk located in the plane $z=0$, with the inner rim at $\rho=a$ and outer rim at $\rho=b$. To introduce globally the potential of the magnetic field $\Phi$, one has to draw mentally a cut along the circle $z=0$, $\rho<a$, on which the potential $\Phi$ experiences a jump of constant value. Then, according to Ampere's theorem, this jump is proportional to the total electric current circulating in the accretion disk.

In the accretion disk, the normal component of the magnetic field should be equal to zero since the magnetic field lines lie in the disk at $z=0, a<\rho<b$, due to its perfect conductivity.

The potential $\Phi$ has the asymptotic of a point-like dipole, and near the coordinate origin $\Phi \approx-A z / r^{3}, A$ being a constant and $r=\sqrt{\rho^{2}+z^{2}}$. Therefore, we can seek the solution in the form

$\Phi=-\frac{A z}{r^{3}}+\varphi$.

The boundary conditions in the plane $z=0$ for the function $\varphi$ are as follows:

$\left.\begin{array}{llc}\varphi=\Phi_{+} & \text {for } & 0<\rho<a \\ \frac{\partial \varphi}{\partial z}=\frac{A}{\rho^{3}} & \text { for } & a<\rho<b \\ \varphi=0 & \text { for } & \rho>b\end{array}\right\}$,

where $\Phi_{+}=(2 \pi / c) J$, and $J$ is the total azimuthal current.

The solution $\varphi$ of Laplace's equation for $z \geq 0$ will be searched for in the form (hereafter $J_{k}(x)$ denotes the Bessel function)

$\varphi=\int_{0}^{\infty} \mathrm{e}^{-k z} J_{0}(k \rho) f(k) \mathrm{d} k$.

Then conditions (2) can be rewritten as

$$
\begin{aligned}
& \int_{0}^{\infty} J_{0}(k \rho) f(k) \mathrm{d} k=\Phi_{+} \quad \text { for } \quad 0<\rho<a, \\
& \int_{0}^{\infty} J_{0}(k \rho) k f(k) \mathrm{d} k=-\frac{A}{\rho^{3}} \text { for } \quad a<\rho<b, \\
& \int_{0}^{\infty} J_{0}(k \rho) f(k) \mathrm{d} k=0 \text { for } \rho>b .
\end{aligned}
$$

These boundary conditions correspond to the triple integral equations, according to the terminology of Sneddon (1966, p. 179). 
For the azimuthal component $\psi$ of the magnetic vector-potential we use the representation (Aly 1980):

$\psi=\int_{0}^{\infty} \mathrm{e}^{-k z} \rho J_{1}(k \rho) f(k) \mathrm{d} k$.

It is convenient to search the function $f(k)$ in the form

$f(k)=\int_{0}^{a} \mu_{1}(s) \sin (k s) \mathrm{d} s+\int_{a}^{b} \mu_{2}(s) \sin (k s) \mathrm{d} s$,

where $\mu_{1}(s)$ and $\mu_{2}(s)$ are two unknown functions. Then condition (6) is satisfied automatically.

Condition (4) can be rewritten in the form

$\int_{\rho}^{a} \frac{\mu_{1}(s) \mathrm{d} s}{\sqrt{s^{2}-\rho^{2}}}+\int_{a}^{b} \frac{\mu_{2}(s) \mathrm{d} s}{\sqrt{s^{2}-\rho^{2}}}=\Phi_{+}$.

Now, let us consider Eq. (5). We first note that the integration by parts in Eq. (8) gives

$\begin{aligned} k f(k)= & \int_{0}^{a} \mu_{1}(s) k \sin (k s) \mathrm{d} s+\int_{a}^{b} \mu_{2}^{\prime}(s) \cos (k s) \mathrm{d} s \\ & +\cos (k a) \mu(a)-\cos (k b) \mu(b) .\end{aligned}$

With this, Eq. (5) assumes the form

$\frac{\partial}{\partial \rho}\left[\int_{0}^{a} \frac{\mu_{1}(s) s \mathrm{~d} s}{\sqrt{\rho^{2}-s^{2}}}+\int_{a}^{\rho} \frac{\mu_{2}(s) s \mathrm{~d} s}{\sqrt{\rho^{2}-s^{2}}}\right]=-\frac{A}{\rho^{2}}$,

and the integration of Eq. (11) finally yields

$\int_{0}^{a} \frac{\mu_{1}(s) s \mathrm{~d} s}{\sqrt{\rho^{2}-s^{2}}}+\int_{a}^{\rho} \frac{\mu_{2}(s) s \mathrm{~d} s}{\sqrt{\rho^{2}-s^{2}}}=\frac{A}{\rho}+$ const.

With the use of the Abel transforms, the system of integral Eqs. (9) and (12), after eliminating the function $\mu_{1}(s)$, reduces to a single Fredholm equation of the second kind.

Introducing the non-dimensional variables

$x \equiv \rho^{2} / a^{2}, \quad t \equiv s^{2} / a^{2}, \quad \beta \equiv b^{2} / a^{2}$,

$\mu_{2}(s)=A y(t) / a^{2}, \quad G=\Phi_{+} a^{2} / A$,

this Fredholm equation for $y(x)$ takes the form

$$
\begin{aligned}
y(x) \sqrt{x-1}= & \frac{2}{\pi x}+C+\frac{2 G}{\pi^{2}}\left(2+\sqrt{x} \ln \frac{\sqrt{x}-1}{\sqrt{x}+1}\right) \\
& +\frac{1}{\pi^{2}} \int_{1}^{\beta} \frac{y(t) \sqrt{t-1} \mathrm{~d} t}{\sqrt{t}(t-x)} \\
& \times\left(\sqrt{t} \ln \frac{\sqrt{t}-1}{\sqrt{t}+1}-\sqrt{x} \ln \frac{\sqrt{x}-1}{\sqrt{x}+1}\right),
\end{aligned}
$$

where $C$ is a non-dimensional constant. Note that on the accretion disk the potential $\varphi$ and the field strength are defined by the following simple formulae:

$\varphi(\rho)=\int_{\rho}^{b} \frac{\mu_{2}(s) \mathrm{d} s}{\sqrt{s^{2}-\rho^{2}}}, \quad B_{\rho}(\rho)=\frac{\partial \varphi}{\partial \rho}$.

Equation (14) has a singular kernel. However, it can be regularized by rewriting it in the form

$$
\begin{gathered}
y(x) \sqrt{x-1}=\frac{2}{\pi x}+C_{1}+\frac{1}{\pi^{2}} \int_{1}^{\beta} \frac{y(t) \mathrm{d} t}{\sqrt{t} \sqrt{t-1}(t-x)} \\
\times\left((t-1) \sqrt{t} \ln \frac{\sqrt{t}-1}{\sqrt{t}+1}-(x-1) \sqrt{x} \ln \frac{\sqrt{x}-1}{\sqrt{t}+1}\right),
\end{gathered}
$$

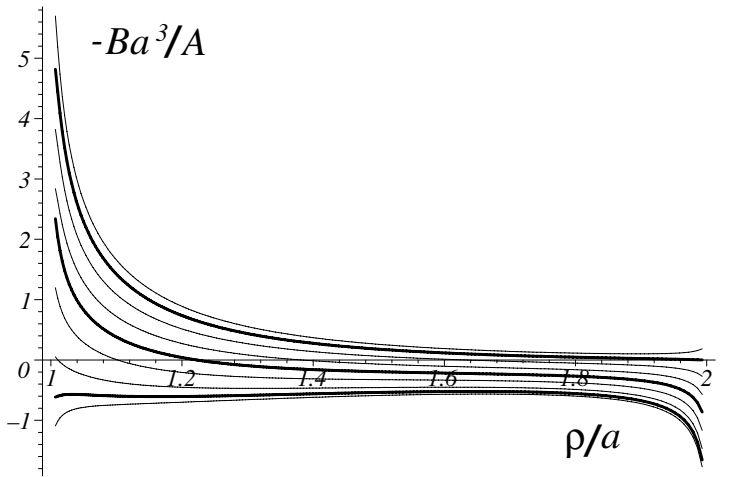

Fig. 1. This figure shows the non-dimensional field strength $B a^{3} / A$ on the accretion disk with the non-dimensional radius $\rho / a$ for $b / a=2$ and different values of the circulation $G$. The central thick curve corresponds to the absence of circulation, $G=0$. The other thick curves correspond to the critical circulation values with a finite magnetic field strength either on the inner or outer edge. The corresponding values of $G$ are equal to -0.4808 and 0.6785 .

where $C_{1} \equiv C+4 G / \pi^{2}$. Here we have used the equality

$\int_{1}^{\beta} \frac{y(t) \mathrm{d} t}{\sqrt{t} \sqrt{t-1}}=2 G$

which follows from Eq. (9). By assigning different values to the constant $C_{1}$, one obtains solutions for different values of the circulation. Using Eq. (17), for each given $C_{1}$ one obtains a corresponding circulation value. Numerical analysis of Eq. (16) makes it possible to construct the magnetic field strength distribution. The field strength distribution along the radius of the disk is shown in Fig. 1 for different circulation values $G=\Phi_{+} a^{2} / A$. The point where $B_{\rho}$ is equal to zero exists only for $-0.4808<G<0.6785$ in the case $b / a=2$. The value of $G=0.6785$ corresponds to minimal magnetic pressure at the outer rim, The value of $G=-0.4808$ corresponds to minimal magnetic pressure on the inner rim.

Using the solution of Eq. (16), one obtains functions $\mu_{1}, \mu_{2}$ in Eq. (8), hence the magnetic field can be constructed. Indeed, from Eqs. (7) and (8) a formula follows suitable for the construction of the magnetic field lines:

$$
\begin{array}{r}
\psi=\frac{A \rho^{2}}{\left(\rho^{2}+z^{2}\right)^{3 / 2}}+\int_{0}^{a} s \mu_{1}(s) \frac{z \mathrm{~d} s}{p^{3} \Delta}+\int_{a}^{b} s \mu_{2}(s) \frac{z \mathrm{~d} s}{p^{3} \Delta} \\
\Delta \equiv \frac{\rho^{2}}{\left(1-p^{2}\right)^{2}}+\frac{z^{2}}{p^{4}}
\end{array}
$$

$p$ denoting the positive root of the equation

$s^{2}=\rho^{2} /\left(1-p^{2}\right)-z^{2} / p^{2}$.

Another way to construct the lines of the magnetic field is to use the formula

$$
\begin{aligned}
\psi(\rho, z) & =\frac{A \rho^{2}}{\left(\rho^{2}+z^{2}\right)^{3 / 2}} \\
& +\frac{\rho}{2 \pi} \int_{a}^{b} \int_{0}^{2 \pi} \frac{B_{\rho}\left(\rho^{\prime}\right) \rho^{\prime} \mathrm{d} \rho^{\prime} \cos \theta \mathrm{d} \theta}{\sqrt{z^{2}+\rho^{2}+\rho^{\prime 2}-2 \rho \rho^{\prime} \cos \theta}},
\end{aligned}
$$

where $B\left(\rho^{\prime}\right)$ is defined by formula (15). $B(\rho)$ can be found after numerical integration of Eqs. (16) and (17). The cross-sections 


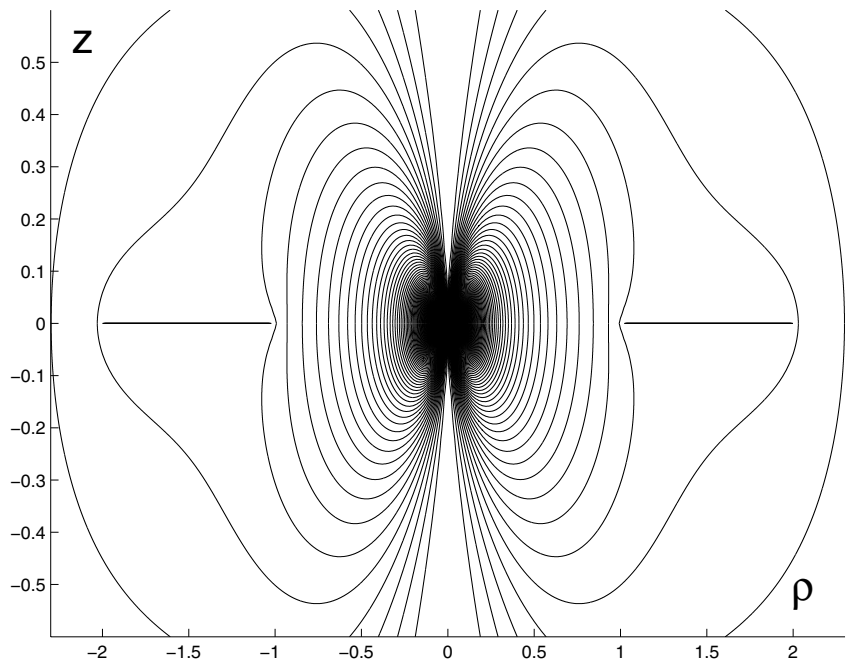

Fig. 2. The cross-section of magnetic surfaces with a plane passing through the symmetry axis in the case $G=0, b / a=2$.

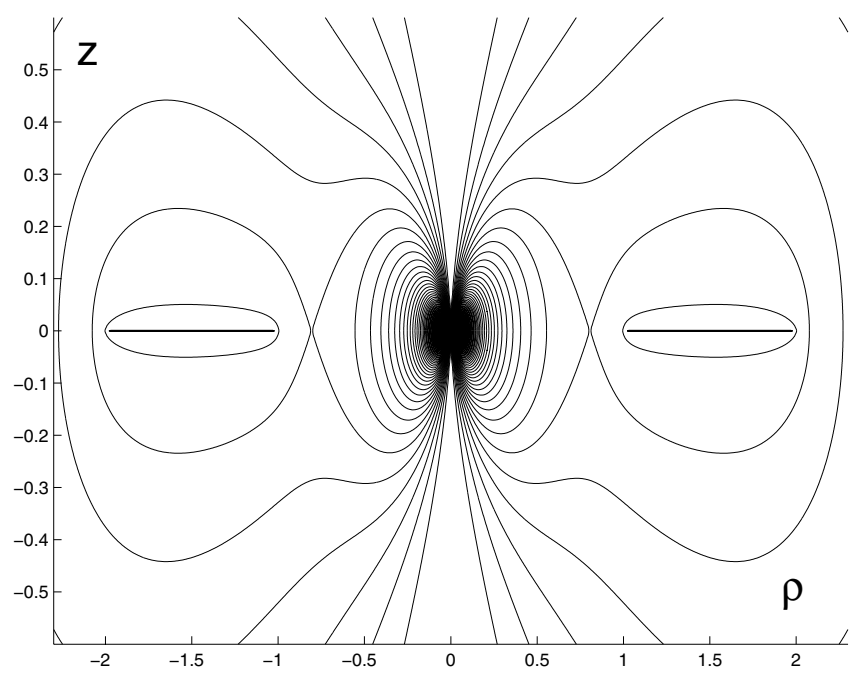

Fig. 3. The cross-section of magnetic surfaces with a plane passing through the symmetry axis in the case $G=-1.59, b / a=2$.

of the spatial magnetic surfaces by a fixed azimuthal angle are given for $b / a=2$ in Figs. 2 and 3 in the cases $G=0, G=$ -1.59 .

As follows from the numerical analysis, there exist critical values of $\Phi_{+}$for which the field strength is bounded either at the inner or outer edge of the accretion disk. For each subcritical value of $\Phi_{+}$there exists a corresponding circumference with radius $\rho_{0}$ on which $B(\rho)$ changes its sign.

To estimate the radius of the magnetosphere in the plane $z=$ 0 , it is necessary to find the distribution of the magnetic field strength $B_{z}$ for $z=0, \quad \rho<a$ (Scharlemann 1978; Aly 1980).

The distribution of the component $B_{z}$ of the magnetic field in the interval $z=0, \quad \rho<a$ within the framework of our approach can be found with the help of the formula

$$
B_{z}=-\frac{A}{\rho^{3}}+\frac{1}{\rho} \frac{\partial}{\partial \rho} \int_{0}^{\rho} \frac{\mu_{1}(s) s \mathrm{~d} s}{\sqrt{\rho^{2}-s^{2}}}
$$

Having expressed $\mu_{1}(s)$ through $\mu_{2}(s)$ and using notations (13), we obtain for $B_{z}$ the formula

$\frac{B_{z} a^{3}}{A}=-\frac{1}{x^{3 / 2}}+\frac{1}{\pi} \frac{\partial}{\partial x} \int_{0}^{x} \frac{\sqrt{s-s^{2}}}{\sqrt{x-t}} \int_{1}^{\beta} \frac{y(t) \mathrm{d} t}{\sqrt{t} \sqrt{t-s} \sqrt{t-1}}$

where $y(x)$ is the numerical solution of Eq. (16).

Here we give a brief summary with a practical description how of to calculate the field. Using the equality $\mu_{2}(s)=$ $A y(t) / a^{2}$ from (13) and substituting the numerical solution $y(x)$ of Eq. (16) into expression (15), we obtain the potential $\varphi(\rho)$ and the field strength $B_{\rho}(\rho)$ on the accretion disk. Formula (20) then makes it possible to obtain the azimuthal component of the magnetic vector potential everywhere in space.

\section{Conclusions}

We have presented an effective method for the construction of the magnetic field around a magnetic dipole surrounded by a finite accretion disc. The field strength distribution in the disk is plotted on the basis of the numerical investigation of the Fredholm equation for different values of the total current (circulation) and ratio $b / a$. The closeness of the disk to the neutron star depends on the value of the total current circulating in the disk. The corresponding maximum and minimum values of the current are found, when the strength of the magnetic field is minimal either on the inner rim of the disk or on the outer rim of the disk. The topological structure of the spatial magnetic surfaces depends drastically on the value of the total azimuthal electric current circulating in the disk.

Acknowledgements. The authors are thankful to the anonymous referee for valuable remarks and suggestions and to Jet Katgert for improving the language of the paper. This research was partially supported by Projects 38495-E, 42729-F from CONACyT of Mexico, by the Russian Foundation for Basic Research, under grants 02-01-00491, 02-01-00613, and NSh-1481.2003.1. N.S. acknowledges financial support from SNI-CONACyT, Mexico.

\section{References}

Aly, J. J. 1980, A\&A, 86, 192

Anzer, U., \& Boerner, G. 1980, A\&A, 83, 133

Börner, G. 1980, Phys. Rep., 60, 152

Ghosh, P., \& Lamb, F. K. 1979, ApJ, 232, 259

Ichimaru, S. 1978, ApJ, 224, 198

Inogamov, N. A., \& Sunyaev, R. A. 1999, Astron. Lett., 25, 269

Kundt, W., \& Robnik, M. 1980, A\&A, 91, 305

Lipunov, V. M. 1978, SvA, 22, 702

Popham, R., \& Narayan, R. 1995, ApJ, 442, 337

Pringle, J. E., \& Rees, M. J. 1972, A\&A, 21, 1

Riffert, H. 1980, Ap\&SS, 71, 195

Scharlemann, E. T. 1978, ApJ, 219, 617

Shakura, N. I., \& Sunyaev, R. A. 1973, A\&A, 24, 337

Sibgatullin, N. R., \& Doroshenko, T. A. 2002, Astron. Lett., 28, 769

Sibgatullin, N. R., \& Sunyaev, R. A. 2000a, Astron. Lett., 26, 699

Sibgatullin, N. R., \& Sunyaev, R. A. 2000b, Astron. Lett., 26, 772

Sneddon, I. N. 1966, Mixed boundary value problems in potential theory (Amsterdam: North-Holland Publishing Co.)

Somov, B. V., Oreshina, A. V., Oreshina, I. V., \& Shakura, N. I. 2003, Adv. Space Res., 32, 1087 